\title{
Magnetoelectric Coupling Based on Protons in Ammonium
}

\section{Sulfate}

\author{
Lei Meng,,$^{1 *}$ Chen $\mathrm{He}^{1 *}$, Fei Yen, ${ }^{1 \dagger}$
}

${ }^{1}$ State Key Laboratory on Tunable Laser Technology, Ministry of Industry and Information Technology Key Laboratory of Micro-Nano Optoelectronic Information

System and the School of Science, Harbin Institute of Technology, Shenzhen, University Town, Shenzhen, Guangdong 518055, P. R. China

\section{Supporting Information:}

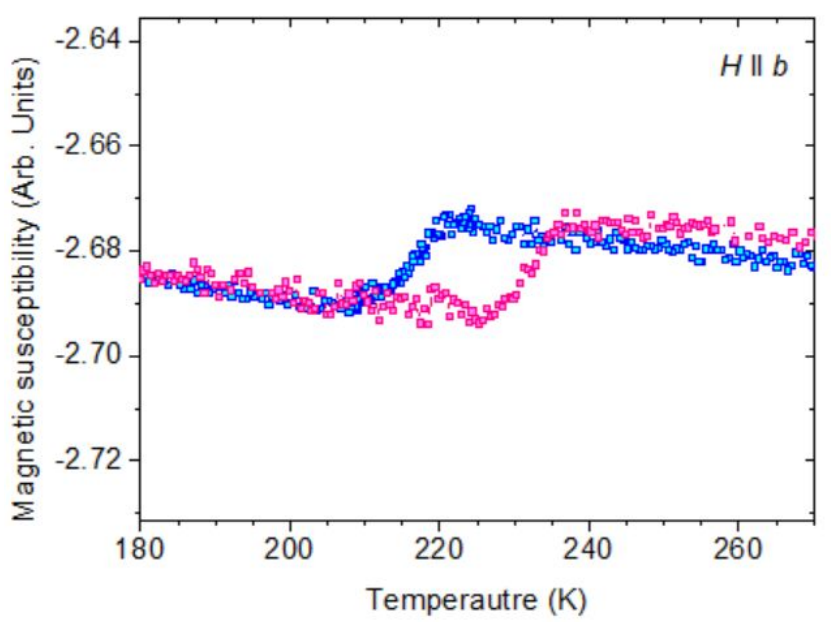

Figure S1. Magnetic susceptibility of an ammonium sulfate $\left(\mathrm{NH}_{4}\right)_{2} \mathrm{SO}_{4}$ crystallite $(2.1$ $\mathrm{mg}$ ) with respect to temperature under $H=10 \mathrm{kOe}$ aligned along the $b$-axis with an MPMS (Magnetic Properties Measurement System) SQuID magnetometer manufactured by Quantum Design, U.S.A. 


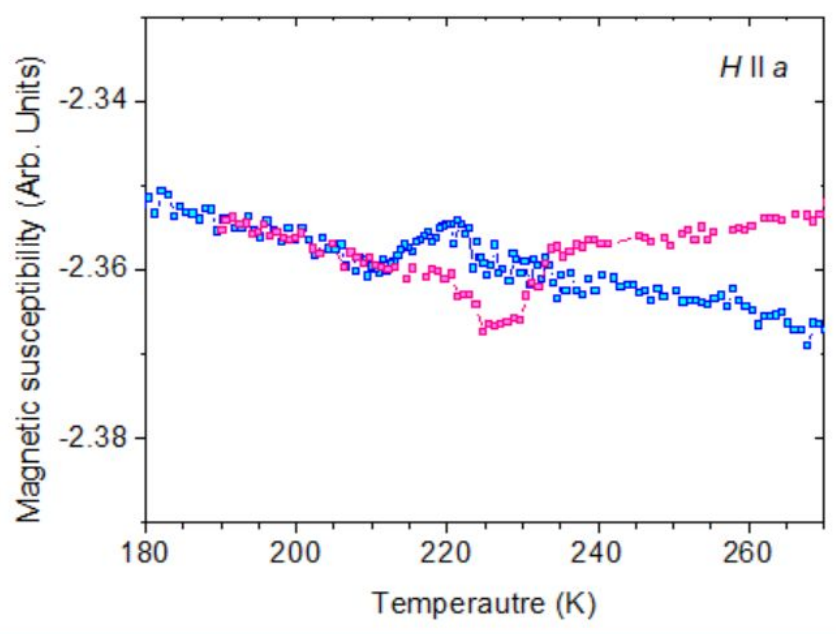

Figure S2. Magnetic susceptibility of the same $\left(\mathrm{NH}_{4}\right)_{2} \mathrm{SO}_{4}$ sample also under $H=10$ kOe but oriented along the $a$-axis.

The obtained results with the MPMS magnetometer were nearly the same. One exception was that the hysteresis regions were larger; this is because the sweeping rates were set to $1.5 \mathrm{~K} / \mathrm{min}$ instead of $0.5 \mathrm{~K} / \mathrm{min}$ when using the PPMS as shown in Figures $3 a, 3 b, 4 a$ and $4 b$. 